\title{
On the optimality of the ideal right-angled 24-cell
}

\author{
ALEXANDER KOLPAKOV
}

\begin{abstract}
We prove that among four-dimensional ideal right-angled hyperbolic polytopes the 24-cell is of minimal volume and of minimal facet number. As a corollary, a dimension bound for ideal right-angled hyperbolic polytopes is obtained.
\end{abstract}

20F55; 52B11, 51M20

\section{Introduction}

In this work, we consider the $24-$ cell $\mathscr{C}$, that is a four-dimensional regular ideal hyperbolic polytope with Schläfli symbol $\{3,4,3\}$ with all dihedral angles right. The polytope $\mathscr{C}$ has 24 octahedral facets, 96 triangular faces, 96 edges and 24 cubical vertex figures. We shall show that it provides a solution to the following problems in the class of ideal right-angled polytopes in $\mathbb{H}^{4}$ :

I Find a polytope of minimal volume,

II Find a polytope of minimal facet number.

Since Coxeter's work [3], the 24-cell is known for its nice combinatorial and geometric structure in the Euclidean sense. We demonstrate that it possesses optimal properties I and II in the hyperbolic setting. Question I is closely related to the volume spectrum of four-dimensional hyperbolic manifolds (see Ratcliffe [10]), question II is new and is both of combinatorial and geometric nature. Furthermore, using the results of Khovanskiı̌ [7] and Nikulin [8], we obtain a new dimension bound for ideal rightangled hyperbolic polytopes. The case of right-angled hyperbolic polytopes with both proper and ideal vertices was considered before by Dufour [6] and by Potyagailo and Vinberg [9].

Acknowledgements This paper is a part of the author's $\mathrm{PhD}$ thesis project supervised by Prof Ruth Kellerhals. The author is grateful to her for inspiration, knowledge and support and to the referee for useful comments and suggestions. This work was supported by the Schweizerischer Nationalfonds SNF grants 200020-121506/1 and 200021-131967/1. 


\section{Preliminaries}

Let $\mathscr{P}$ be a polytope in the hyperbolic $n$-dimensional space $\mathbb{H}^{n}$, that means $\mathscr{P}=$ $\bigcap_{i \in I} H_{i}^{-},|I|<\infty$, with finitely many half-spaces $H_{i}^{-} \subset \mathbb{H}^{n}$ enclosed by the respective hyperplanes $H_{i}$. In particular, $\mathscr{P}$ is convex. The part of the polytope $\mathscr{P}$ contained in such a hyperplane is called a facet and has dimension $n-1$. We define the low-dimensional faces of $\mathscr{P}$ by induction as non-empty intersections of the higherdimensional ones. Let us denote the set of $k$-dimensional faces by $\Omega_{k}(\mathscr{P}), 0 \leq k<n$, $\Omega_{n}(\mathscr{P}):=\mathscr{P}$. We call the $k$-dimensional faces of $\mathscr{P}$ for $k=0$ and 1 , its vertices and edges, respectively. Let $\mathrm{f}_{k}$ denote the number of $k$-dimensional faces and let $\mathbf{f}(\mathscr{P})=\left(\mathrm{f}_{0}, \ldots, \mathrm{f}_{n-1}\right)$ be the face vector of the polytope $\mathscr{P}$.

The faces of a given $n$-polytope $\mathscr{P}$ form a lattice $\Omega(\mathscr{P}):=\bigcup_{k=0}^{n} \Omega_{k}(\mathscr{P})$ ordered by the set-theoretical face inclusion. Call two polytopes $\mathscr{P}_{1}$ and $\mathscr{P}_{2}$ combinatorially isomorphic if their face lattices $\Omega\left(\mathscr{P}_{1}\right)$ and $\Omega\left(\mathscr{P}_{2}\right)$ are isomorphic. Call $\mathscr{P} \subset \mathbb{H}^{n}$ a regular hyperbolic polytope if it is combinatorially isomorphic to a regular $n$-dimensional Euclidean polytope and all the dihedral angles of $\mathscr{P}$ in its co-dimension two faces are equal. Recall that there are infinitely many regular polygons. Dimension three provides five Platonic solids. There exist six regular four-dimensional polytopes. Starting from dimension five, there are only three combinatorial types of convex regular polytopes (see Coxeter [3, Table I]).

A polytope is called non-obtuse if all the dihedral angles in its co-dimension two faces are not greater than $\pi / 2$. A polytope is right-angled if all these dihedral angles equal $\pi / 2$. Call a hyperbolic polytope $\mathscr{P}$ ideal if all its vertices are ideal points of $\mathbb{H}^{n}$, that is, all of them belong to $\partial \overline{\mathbb{H}}^{n}$. A polytope $\mathscr{P} \subset \mathbb{H}^{n}$ is simple if each vertex belongs to $n$ facets only, and $\mathscr{P}$ is called simple at edges if each edge belongs to $n-1$ facets only. An infinitesimal link (w.r.t. the Euclidean metric on $\overline{\mathbb{H}}^{n}$ ) of a vertex of a polytope is called its vertex figure (w.r.t. the given vertex). Every vertex figure of a compact non-obtuse hyperbolic polytope is a combinatorial simplex of co-dimension one (see Vinberg [11, page 108, Theorem 1.8]). Every vertex figure of an ideal right-angled hyperbolic polytope is a combinatorial cube of co-dimension one (see Dufour [6, Proposition 1]). Thus, a compact non-obtuse hyperbolic polytope is simple and an ideal right-angled hyperbolic polytope is simple at edges.

The 24-cell considered as a regular polytope has the Schläfli symbol $\{3,4,3\}$, octahedral facets $\{3,4\}$ and cubical vertex figures $\{4,3\}$. Note that this is the only regular four-dimensional polytope having each vertex figure a cube (see Coxeter [3, Table I]) and thus realisable as an ideal right-angled hyperbolic one. We denote it by $\mathscr{C}$ and call the hyperbolic 24-cell. 


\section{The 24-cell and volume minimality}

Lemma 1 (Combinatorial identities) Let $\mathscr{P} \subset \mathbb{H}^{4}$ be an ideal right-angled polytope with face vector $\mathbf{f}(\mathscr{P})=\left(\mathrm{f}_{0}, \mathrm{f}_{1}, \mathrm{f}_{2}, \mathrm{f}_{3}\right)$. Then the following combinatorial identities hold.

$$
\begin{aligned}
& \mathrm{f}_{0}-\mathrm{f}_{1}+\mathrm{f}_{2}-\mathrm{f}_{3}=0, \\
& \mathrm{f}_{1}=4 \mathrm{f}_{0}, \\
& 12 \mathrm{f}_{0}=\sum_{F \in \Omega_{2}(\mathscr{P})} \mathrm{f}_{0}(F) .
\end{aligned}
$$

Proof We list the proofs of (1)-(3) below in the respective order.

(1) This is Euler's identity. Since $\mathscr{P}$ is a convex four-dimensional polytope, its surface $\partial \mathscr{P}$ is homeomorphic to $\mathbb{S}^{3}$. Hence, for the Euler characteristic of $\partial \mathscr{P}$, we have $\mathrm{f}_{0}-\mathrm{f}_{1}+\mathrm{f}_{2}-\mathrm{f}_{3}=: \chi(\partial \mathscr{P})=\chi\left(\mathbb{S}^{3}\right)=0$.

(2) Let $v \in \Omega_{0}(\mathscr{P})$ be a vertex. Then each vertex figure $\mathscr{P}_{v}$ is a cube, since $\mathscr{P}$ is an ideal right-angled polytope (see Dufour [6, Proposition 1]). The vertices of $\mathscr{P}_{v}$ correspond to the edges of $\mathscr{P}$ emanating from a given vertex $v \in \Omega_{0}(\mathscr{P})$. This means that eight edges are adjacent at $v$. On the other hand, each edge has two vertices. Thus, we obtain $2 \mathrm{f}_{1}=8 \mathrm{f}_{0}$ and (2) follows.

(3) The edges of the vertex figure $\mathscr{P}_{v}$ correspond to the two-dimensional faces of $\mathscr{P}$ meeting $v$. Thus, twelve two-dimensional faces meet at each vertex. Hence, if we sum up the number of vertices $\mathrm{f}_{0}(F)$ over all the two-dimensional faces $F \in \Omega_{2}(\mathscr{P})$, we count each vertex of $\mathscr{P}$ twelve times. Then the desired formula follows and the lemma is proven.

Lemma 2 (Volume formula) Let $\mathscr{P} \subset \mathbb{H}^{4}$ be an ideal right-angled polytope with face vector $\mathbf{f}(\mathscr{P})=\left(\mathrm{f}_{0}, \mathrm{f}_{1}, \mathrm{f}_{2}, \mathrm{f}_{3}\right)$. Then its volume equals

$$
\operatorname{Vol} \mathscr{P}=\frac{\mathrm{f}_{0}-\mathrm{f}_{3}+4}{3} \pi^{2} .
$$

Proof Let $G$ be the group generated by reflections in the supporting hyperplanes of the facets of $\mathscr{P}$. The group $\mathscr{G}$ is a Coxeter group acting discretely on $\mathbb{H}^{4}$ with Poincaré polytope $\mathscr{P}$. Hence $\operatorname{Vol} \mathscr{P}=\operatorname{coVol} \mathscr{G}$. By Zehrt [12, Corollary 4.2], we obtain

$$
\operatorname{coVol} \mathscr{G}=\frac{\pi^{2}}{3}\left(\kappa(\mathscr{P})-2 \sum_{F \in \Omega_{2}(\mathscr{P})} \frac{\mathrm{f}_{0}(F)-2}{|\operatorname{Stab}(F)|}+4 \sum_{v \in \Omega_{0}(\mathscr{P})} \frac{1}{|\operatorname{Stab}(v)|}\right),
$$


where $\kappa(\mathscr{P}):=4-2\left(\mathrm{f}_{0}+\mathrm{f}_{2}\right)+\sum_{F \in \Omega_{2}(\mathscr{P})} \mathrm{f}_{0}(F)$. Here $\operatorname{Stab}(\star) \leqslant \varphi$ means the stabilizer of $\star$, where $\star$ is a two-dimensional face $F$ or a vertex $v$ of $\mathscr{P}$.

For each $F \in \Omega_{2}(\mathscr{P})$ the group $\operatorname{Stab}(F)$ is generated by two reflections in the facets meeting at $F$. Since $\mathscr{P}$ is a right-angled polytope, the group $\operatorname{Stab}(F)$ is generated by reflections in two orthogonal mirrors. Thus, $\operatorname{Stab}(F) \simeq D_{2}$, the dihedral group of order four. Each vertex $v \in \Omega_{0}(\mathscr{P})$ is ideal, that is, belongs to $\partial \overline{\mathbb{H}}^{4}$, so the stabilizer $\operatorname{Stab}(v)$ is a Coxeter group generated by reflection in the faces of a Euclidean cube. Hence $\operatorname{Stab}(v)$ is infinite.

We obtain the desired volume formula by substituting the values $|\operatorname{Stab}(F)|=4$ and $|\operatorname{Stab}(v)|=\infty$ into (4), and by applying the formulas of Lemma 1 to the computation.

The hyperbolic 24-cell $\mathscr{C}$ has $\mathrm{f}_{0}=\mathrm{f}_{3}=24, \mathrm{f}_{1}=\mathrm{f}_{2}=96$, see Coxeter [3, Table I, (ii)]. Hence, by the lemma above, its volume equals $4 \pi^{2} / 3$.

Theorem 3 (Minimal volume) A four-dimensional ideal right-angled hyperbolic polytope of minimal volume is $\mathscr{C}$, up to an isometry.

Proof Let us consider an ideal right-angled hyperbolic polytope $\mathscr{P} \subset \mathbb{H}^{4}$. Let $\mathrm{f}_{2}(k)$ denote the number of its two-dimensional $k$-gonal faces, $k \geq 3$, which are ideal hyperbolic polygons. Then

$$
\mathrm{f}_{2}=\mathrm{f}_{2}(3)+\cdots+\mathrm{f}_{2}(N),
$$

where $N=\max _{F \in \Omega_{2}(\mathscr{P})} \mathrm{f}_{0}(F) \geq 3$. By Lemma 1, formula (3), we obtain

$$
12 \mathrm{f}_{0}=\sum_{F \in \Omega_{2}(\mathscr{P})} \mathrm{f}_{0}(F)=3 \mathrm{f}_{2}(3)+\cdots+N \mathrm{f}_{2}(N) .
$$

By using Lemma 1, formulas (1)-(2), one subsequently computes

$$
\mathrm{f}_{0}-\mathrm{f}_{3}=4 \mathrm{f}_{0}-\mathrm{f}_{2}=\frac{1}{3} \sum_{k=4}^{N}(k-3) \mathrm{f}_{2}(k) \geq 0 .
$$

Then, by Lemma 2,

$$
\operatorname{Vol} \mathscr{P} \geq \frac{4}{3} \pi^{2}=\operatorname{Vol} \mathscr{C} .
$$

If $\operatorname{Vol} \mathscr{P}$ equals the volume of $\mathscr{C}$, one immediately has $\mathrm{f}_{2}(k)=0$ for all $k \geq 4$ by (5). This means that all the two-dimensional faces of $\mathscr{P}$ are triangles. Consider a facet $P \in \Omega_{3}(\mathscr{P})$. Observe that $P \subset \mathbb{H}^{3}$ is an ideal right-angled polyhedron which has only triangular faces. Then $P$ is a combinatorial octahedron and it is isometric to the 
right-angled hyperbolic octahedron by work of Andreev [1, Theorem 3; 2, Theorem 2]. Hence, all the facets of $\mathscr{P}$ are ideal right-angled octahedra. So the polytope $\mathscr{P}$ is combinatorially isomorphic to a regular four-dimensional Euclidean polytope with octahedral facets only, that is, the $24-$ cell by Coxeter [3, Table I, (ii)]. Thus $\mathscr{P}$ is isometric to $\mathscr{C}$ by Andreev's theorem [1, Theorem 3].

\section{The 24-cell and facet number minimality}

Theorem 4 (Minimal facet number) The facet number of a four-dimensional ideal right-angled hyperbolic polytope $\mathscr{P}$ satisfies the inequality $\mathrm{f}_{3}(\mathscr{P}) \geq \mathrm{f}_{3}(\mathscr{C})=24$. Any four-dimensional ideal right-angled hyperbolic polytope $\mathscr{P}$ with $\mathrm{f}_{3}(\mathscr{P})=24$ is isometric to the hyperbolic $24-$ cell $\mathscr{C}$.

The proof will be based on Proposition 5 and Lemma 6 below. Their proofs will be given in Section 3.3.

\subsection{Three-dimensional ideal right-angled hyperbolic polyhedra with few faces}

Let $\mathscr{A}_{k} \subset \mathbb{H}^{3}, k \geq 3$, be an ideal right-angled antiprism depicted in Figure 1. In the figure, the leftmost and the rightmost edges are identified, so that the surface of the polyhedron is partitioned into top and bottom $k$-gonal faces and $2 k$ triangular faces in the annulus between them. Such an antiprism exists for every $k \geq 3$ and it is unique up to an isometry due to Andreev [1, Theorem 3; 2, Theorem 2].

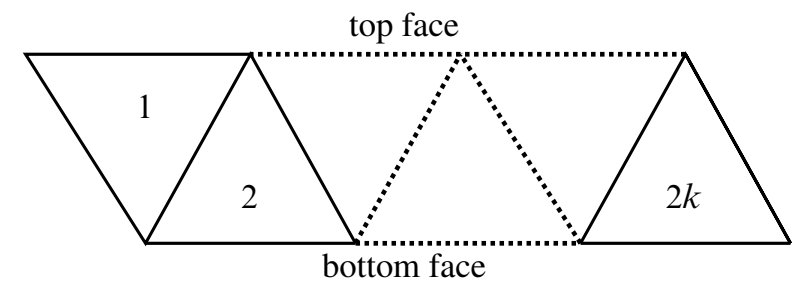

Figure 1: Antiprism $\mathscr{A}_{k}, k \geq 3$

Antiprisms $\mathscr{A}_{k}$ will later play the rôle of possible facets for a four-dimensional ideal right-angled hyperbolic polytope in the proof of Theorem 4.

Proposition 5 (Antiprism's optimality) A three-dimensional ideal right-angled hyperbolic polyhedron of minimal facet number, which has at least one $k$-gonal face, $k \geq 3$, is isometric to the antiprism $\mathscr{A}_{k}$ with $\mathrm{f}_{2}\left(\mathscr{A}_{k}\right)=2 k+2$. 
Proof Let $\mathscr{P} \subset \mathbb{H}^{3}$ be an ideal right-angled polyhedron. Let $F \in \Omega_{2}(\mathscr{P})$ be a $k$-gonal face, $k \geq 3$. For each edge $e \in \Omega_{1}(F)$ there is exactly one further face adjacent to $F$ along $e$. For each vertex $v$, being four-valent by Andreev's theorem [2, Theorem 2], there exists a face intersecting $F$ at $v$ only. Moreover, all the faces mentioned above are different from each other, so that we have $\mathrm{f}_{2}(\mathscr{P}) \geq 2 k+1$. Observe that these faces can not constitute yet a polyhedron. Indeed, consider $F$ as a "bottom" face of $\mathscr{P}$. Then the new faces we have added make a surface wrapping around the interior of $\mathscr{P}$ along the edges of $F$. Since all vertices are four-valent, at least one additional "top" face is required to close up the polyhedron. Hence $\mathrm{f}_{2}(\mathscr{P}) \geq 2 k+2$. The antiprism $\mathscr{A}_{k}$ satisfies

$$
\mathrm{f}_{2}\left(\mathscr{A}_{k}\right)=2 k+2
$$

and so has minimal facet number.

It remains to show that a polyhedron $\mathscr{P}$ with $\mathrm{f}_{2}(\mathscr{P})=\mathrm{f}_{2}\left(\mathscr{A}_{k}\right)$ is in fact isometric to $\mathscr{A}_{k}$. Since $\mathscr{P}$ has four-valent vertices, $2 \mathrm{f}_{1}(\mathscr{P})=4 \mathrm{f}_{0}(\mathscr{P})$. From this equality and Euler's identity $\mathrm{f}_{0}(\mathscr{P})-\mathrm{f}_{1}(\mathscr{P})+\mathrm{f}_{2}(\mathscr{P})=2$ we obtain that

$$
\mathrm{f}_{2}(\mathscr{P})=\mathrm{f}_{0}(\mathscr{P})+2 .
$$

Consider the faces adjacent to the $k$-gon $F$ along its edges. We shall prove that no pair of them can have a common vertex $v \notin \Omega_{0}(F)$. By supposing the contrary, let us denote two such faces $F_{i}, i=1,2$, and let them intersect at $v$. Observe that $F_{i}$, $i=1,2$, are adjacent to $F$ along two disjoint edges $e_{1}$ and $e_{2}$. In fact, if $e_{1}$ intersects $e_{2}$ in a vertex $u \in \Omega_{0}(F)$, then since $\mathscr{P}$ has convex faces we obtain two geodesic segments joining $v$ to $u$. One of them belongs to $F_{1}$ and the other belongs to $F_{2}$. This is impossible, unless the considered segments are represented by a common edge $e$ of $F_{i}, i=1,2$, adjacent to both $v$ and $u$. But then the vertex $u$ has only three adjacent edges: $e_{1}, e_{2}$ and $e$. This is a contradiction to $u$ having valency four. Now if $F_{1}$ and $F_{2}$ share an edge $e$ such that $v \in \Omega_{0}(e)$, then condition $\left(\mathfrak{m}_{2}\right)$ of Andreev's theorem [2, Theorem 2] does not hold as depicted in Figure 2. If $F_{1}$ and $F_{2}$ share only the vertex $v$, then condition $\left(\mathfrak{m}_{5}\right)$ of [2, Theorem 2] is not satisfied as depicted in Figure 3.

Suppose that a face $F^{\prime}$ adjacent to the $k$-gon $F \in \Omega_{2}(\mathscr{P})$ along an edge is not triangular. Then $F^{\prime}$ has $\mathrm{f}_{0}\left(F^{\prime}\right)$ vertices, and two among them are already counted in $\mathrm{f}_{0}(F)$. Hence we have at least

$$
\sum_{\substack{F^{\prime} \text { adjacent to } \\ F \text { along an edge }}}\left(\mathrm{f}_{0}\left(F^{\prime}\right)-2\right) \geq(k-1)+2=k+1
$$




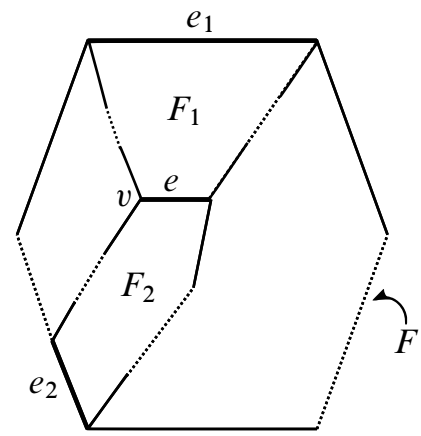

Figure 2: Three-circuit deprecated by Andreev's theorem consists of the faces $F, F_{1}$ and $F_{2}$

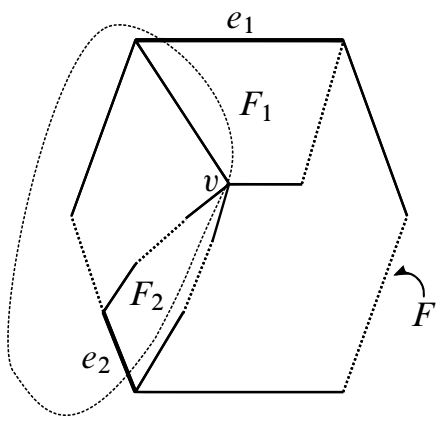

Figure 3: The circuit deprecated by Andreev's theorem is indicated by the dashed line

additional vertices, since $\mathrm{f}_{0}\left(F^{\prime}\right) \geq 3$ for each $F^{\prime}$ among $k$ faces adjacent to $F$ and at least one such face $F^{\prime}$ has $\mathrm{f}_{0}\left(F^{\prime}\right) \geq 4$. Thus $\mathrm{f}_{0}(\mathscr{P}) \geq 2 k+1$, and by (7) the estimate $\mathrm{f}_{2}(\mathscr{P}) \geq 2 k+3$ follows. Equality (6) implies $\mathrm{f}_{2}(\mathscr{P})>\mathrm{f}_{2}\left(\mathscr{A}_{k}\right)$ and we arrive at a contradiction. Hence all the faces adjacent to $F$ along its edges are triangular.

Consider the faces of $\mathscr{P}$ adjacent to the $k$-gon $F \in \Omega_{2}(\mathscr{P})$ only at its vertices. Suppose that one of them, say $F^{\prime}$, is not triangular. Then we have

$$
\sum_{\substack{F^{\prime} \text { adjacent to } \\ F \text { at a vertex }}} \mathrm{f}_{1}\left(F^{\prime}\right) \geq 3(k-1)+4=3 k+1
$$

additional edges. But then $\mathrm{f}_{1}(\mathscr{P}) \geq 4 k+1$ and we arrive at a contradiction. Indeed, in this case $\mathrm{f}_{1}(\mathscr{P})>\mathrm{f}_{1}\left(\mathscr{A}_{k}\right)=4 k$, while a polyhedron of minimal facet number has all $\mathrm{f}_{i}$ minimal, $i=0,1,2$, as follows from equation (7) and the remarks before.

Hence we have a $k$-gonal face $F, k \geq 3$, together with $2 k$ triangular side faces adjacent to it along the edges and at the vertices. By adding another one $k$-gonal face 
we close up the polyhedron $\mathscr{P}$, while its vertex number remains unchanged. Observe that there is no other way to finish this construction without increasing the vertex number.

Thus, an ideal right-angled polyhedron $\mathscr{P} \subset \mathbb{H}^{3}$ having minimal face number, which contains at least one $k$-gon, is combinatorially isomorphic to $\mathscr{A}_{k}$. By work of Andreev [1, Theorem $3 ; 2$, Theorem 2], the polyhedron $\mathscr{P}$ is isometric to $\mathscr{A}_{k}$.

Note (to Proposition 5) The classification of polygonal maps on the two-dimensional sphere given by Deza, Sikirić and Shtogrin [5] provides another argument to show the uniqueness of antiprism stated above. Namely, [5, Theorem 1] says that $\mathscr{P}$ has in fact not less than two $k$-gonal faces. Hence $\mathrm{f}_{2}(\mathscr{P})=2 k+2$ if and only if $\mathscr{P}$ has exactly two $k$-gonal faces and $2 k$ triangular faces. Polygonal maps of this kind are classified by [5, Theorem 2]. Among them only the map isomorphic to the one-skeleton of $\mathscr{A}_{k}$ satisfies Steiniz's theorem (see Ziegler [13, Chapter 4]). Thus, the polyhedron $\mathscr{P}$ is combinatorially isomorphic to $\mathscr{A}_{k} \cdot{ }^{1}$

\subsection{Combinatorial constraints on facet adjacency}

Let $F_{1}, \ldots, F_{m}$ be an ordered sequence of facets of a given hyperbolic polytope $\mathscr{P} \subset \mathbb{H}^{4}$ such that each facet is adjacent only to the previous and the following ones either through a co-dimension two face or through an ideal vertex, while the last facet $F_{m}$ is adjacent only to the first facet $F_{1}$ (through a co-dimension two face or through an ideal vertex, as before) and no three of them share a lower-dimensional face. Call the sequence $F_{1}, \ldots, F_{m} a(k, \ell)$ circuit, $k+\ell=m$, if it comprises $k$ co-dimension two faces and $\ell$ ideal vertices shared by the facets. We complete the analysis carried out by Potyagailo and Vinberg [9] in the following way.

Lemma 6 (Adjacency constraints) Let $\mathscr{P} \subset \mathbb{H}^{4}$ be an ideal right-angled polytope. Then $\mathscr{P}$ contains no $(3,0),(4,0)$ and $(2,1)$ circuits.

Proof By [9, Proposition 4.1] there are no $(3,0)$ and $(2,1)$ circuits. Suppose on the contrary that there exists a $(4,0)$ circuit formed by the facets $F_{k} \in \Omega_{3}(\mathscr{P}), k=1,2,3,4$. Let $e_{k}, k=1,2,3,4$, denote the outer unit vector normal to the support hyperplane of $F_{k}$. Consider the Gram matrix of these vectors w.r.t. the Lorentzian form $\langle\cdot, \cdot\rangle_{4,1}$ :

$$
G=\left(\left\langle e_{i}, e_{j}\right\rangle\right)_{i, j=1}^{4}=\left(\begin{array}{cccc}
1 & 0 & -\cosh \rho_{13} & 0 \\
0 & 1 & 0 & -\cosh \rho_{24} \\
-\cosh \rho_{13} & 0 & 1 & 0 \\
0 & -\cosh \rho_{24} & 0 & 1
\end{array}\right)
$$

\footnotetext{
${ }^{1}$ The author is grateful to Michel Deza for indicating the very recent paper [5].
} 
where $\rho_{i j}>0$ is the length of the common perpendicular between two disjoint support hyperplanes for $F_{i}$ and $F_{j}$ respectively. The eigenvalues of $G$ are $\left\{1 \pm \cosh \rho_{13}, 1 \pm\right.$ $\left.\cosh \rho_{24}\right\}$, that means two of them are strictly negative and two are strictly positive. Thus, we arrive at a contradiction with the signature of a Lorentzian form.

\subsection{Proof of Theorem 4}

Let $\mathscr{P} \subset \mathbb{H}^{4}$ be an ideal right-angled polytope. Let $P \in \Omega_{3}(\mathscr{P})$ be a facet. For every two-face $F \in \Omega_{2}(P)$ there exists a corresponding facet $P^{\prime} \in \Omega_{3}(\mathscr{P}), P^{\prime} \neq P$, such that $P$ and $P^{\prime}$ share the face $F$. Since each vertex figure of $\mathscr{P}$ is a cube, there exists a respective facet $P^{\prime \prime} \in \Omega_{3}(\mathscr{P})$ for every vertex $v \in \Omega_{0}(P)$. The vertex figure is depicted in Figure 4, where the grey bottom face of the cube corresponds to $P$ and the top face corresponds to $P^{\prime \prime}$. These new facets $P^{\prime}$ and $P^{\prime \prime}$ together with $P$ are pairwise different. In order to show this we use the following convexity argument.

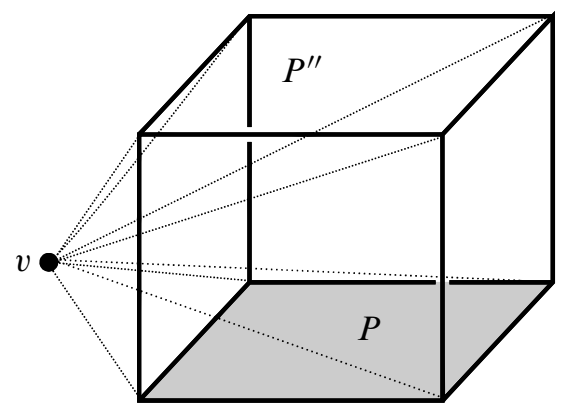

Figure 4: The vertex figure $\mathscr{P}_{v}$

Convexity argument First, observe that no facet of a convex polytope can meet another one at two different two-faces. Now suppose that $P^{\prime} \in \Omega_{3}(\mathscr{P})$ is a facet adjacent to $P$ at a face $F \in \Omega_{2}(P)$ and a single vertex $v \in \Omega_{0}(P)$ not in $F$. The facets $P$ and $P^{\prime}$ have non-intersecting interiors, but the geodesic going through a given point of $F$ to $v$ belongs to both of them by the convexity of $\mathscr{P}$. So we arrive at a contradiction.

The same contradiction arises if we suppose that there is a facet $P^{\prime} \in \Omega_{3}(\mathscr{P})$ adjacent to $P$ at two distinct vertices $v, v^{\prime} \in \Omega_{0}(P)$. In this case we consider the geodesic in $P$ going through $v$ to $v^{\prime}$.

By the convexity argument above, the facet number of $\mathscr{P}$ has the lower bound

$$
\mathrm{f}_{3}(\mathscr{P}) \geq \mathrm{f}_{2}(P)+\mathrm{f}_{0}(P)+1
$$


or, by means of equality (7),

$$
\mathrm{f}_{3}(\mathscr{P}) \geq 2 \mathrm{f}_{2}(P)-1
$$

Observe that the hyperbolic $24-$ cell $\mathscr{C}$ has only triangle two-faces. Suppose that $\mathscr{P}$ has at least one $k$-gonal face $F \in \Omega_{2}(\mathscr{P})$ with $k \geq 4$. We shall show that the estimate $\mathrm{f}_{3}(\mathscr{P}) \geq 25$ holds, by considering several cases as follows.

Case A Suppose that $\mathscr{P}$ has a $k$-gonal two-dimensional face with $k \geq 6$. Then, by (8) and Proposition 5, we have

$$
\mathrm{f}_{3}(\mathscr{P}) \geq 2 \mathrm{f}_{2}\left(\mathscr{A}_{k}\right)-1=2(2 k+2)-1 \geq 27 .
$$

Thus $\mathscr{P}$ is not of minimal facet number.

Case B Suppose that $\mathscr{P}$ has a pentagonal two-dimensional face $F$ contained in a facet $P \in \Omega_{3}(\mathscr{P})$. Suppose $P$ is not isometric to $\mathscr{A}_{5}$. This assumption implies $\mathrm{f}_{2}(P)>12$. Then $(8)$ grants $\mathrm{f}_{3}(\mathscr{P}) \geq 25$.

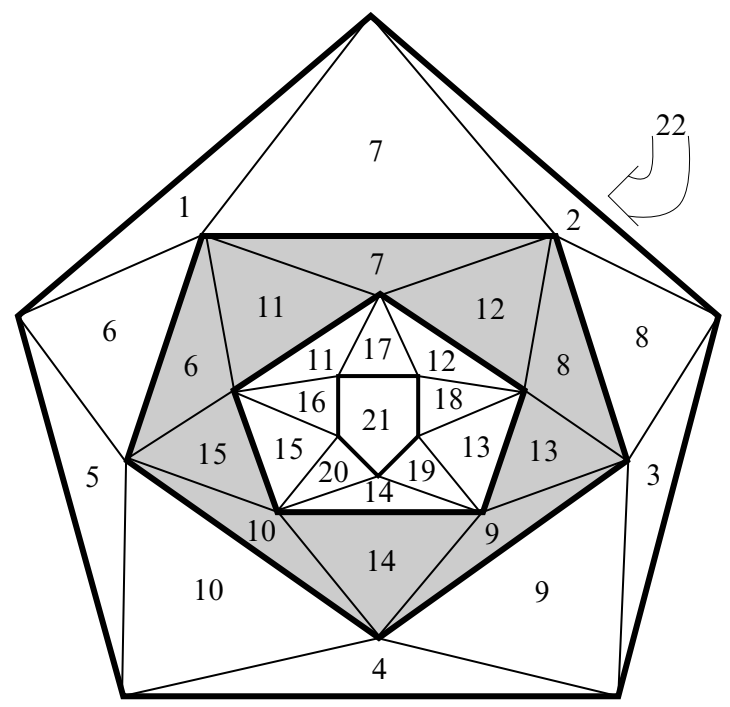

Figure 5: Three facets of $\mathscr{P}$ isometric to $\mathscr{A}_{5}$ and their neighbours

Case C Suppose that all the facets of $\mathscr{P}$ containing a pentagonal two-face are isometric to $\mathscr{A}_{5}$. Let $P_{0}$ be one of them. Then it has two neighbouring facets $P_{k}, k=1,2$ both isometric to $\mathscr{A}_{5}$. Now we count the facets adjacent to $P_{k}, k=0,1,2$ in Figure 5, where $P_{0}$ is coloured grey. Observe that two-faces in Figure 5 sharing an edge are 
marked with the same number and belong to a common facet, since $\mathscr{P}$ is simple at edges. However, the two-faces marked with different numbers, correspond to different adjacent facets. Suppose on the contrary that there are two faces $F \in \Omega_{2}\left(P_{i}\right), F^{\prime} \in \Omega_{2}\left(P_{j}\right)$, $i, j \in\{0,1,2\}$, marked with distinct numbers and a facet $P^{\prime} \in \Omega_{3}(\mathscr{P})$ such that $P^{\prime}$ is adjacent to $P_{i}$ at $F$ and to $P_{j}$ at $F^{\prime}$ and consider the following cases.

Case C1 If $i=j$, we arrive at a contradiction by the convexity argument above.

Case C2 If $i=0, j \in\{1,2\}$, then there exists a unique geodesic joining a point $p$ of $F$ to a point $p^{\prime}$ of $F^{\prime}$. Observe in Figure 5, that the point $p^{\prime}$ may be chosen so that $p^{\prime} \in F^{\prime} \cap P_{0}$. Then the geodesic between $p$ and $p^{\prime}$ intersects both the interior of $P^{\prime}$ and the interior of $P_{0}$. Again, we use the convexity argument and arrive at a contradiction.

Case $\mathbf{C 3}$ Let $i=1, j=2$. Then if there exist a face $\widetilde{F} \in \Omega_{2}\left(P_{0}\right), \tilde{F} \cap F \neq \varnothing$, and a face $\widetilde{F}^{\prime} \in \Omega_{2}\left(P_{0}\right), \widetilde{F}^{\prime} \cap F^{\prime} \neq \varnothing$, we reduce our argument to case $\mathrm{C} 1$ by considering a geodesic segment joining a point of $\widetilde{F} \cap F$ to a point of $\widetilde{F}^{\prime} \cap F^{\prime}$.

The only case when no such two faces $\widetilde{F}$ and $\widetilde{F}^{\prime}$ exist is if $F$ has number 21 and $F^{\prime}$ has number 22 in Figure 5. Then the $(4,0)$-circuit $P_{0} P_{1} P^{\prime} P_{2}$ appears, in contrary to Lemma 6.

Thus, one has 22 new facets adjacent to $P_{k}, k=0,1,2$. Together with $P_{k}$ themselves, $k=0,1,2$, they provide $\mathrm{f}_{3}(\mathscr{P}) \geq 25$.
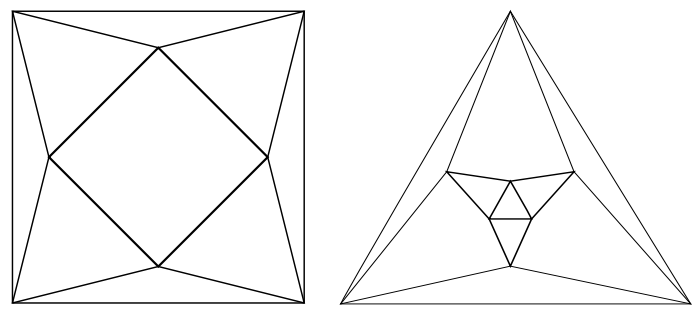

Figure 6: Hyperbolic octahedrites with 8 (left) and 9 (right) vertices

Case D By now, cases A, B and C imply that if an ideal right-angled hyperbolic polytope $\mathscr{P} \subset \mathbb{H}^{4}$ has at least one $k$-gonal face with $k \geq 5$, then $\mathrm{f}_{3}(\mathscr{P}) \geq 25$. Suppose that $\Omega_{2}(\mathscr{P})$ contains only triangles and quadrilaterals.

By Andreev's theorem [2], each facet $P \in \Omega_{3}(\mathscr{P})$ has only four-valent vertices. By assumption, $P$ has only triangular and quadrilateral faces. Combinatorial polyhedra of this type are introduced by Deza and Shtogrin [4] as octahedrites and the list 
of those possessing up to 17 vertices is given. Note that in view of (8) we may consider octahedrites that have not more than twelve faces or, by equality (7) from Proposition 5, ten vertices. In Figure 6, 7 we depict only those realisable as ideal right-angled hyperbolic polyhedra with eight, nine and ten vertices. The ideal rightangled octahedron has six vertices and completes the list. By considering each of the polyhedra in Figure 6 and Figure 7 as a possible facet $P \in \Omega_{3}(\mathscr{P})$, we shall derive the estimate $\mathrm{f}_{3}(\mathscr{P}) \geq 25$.

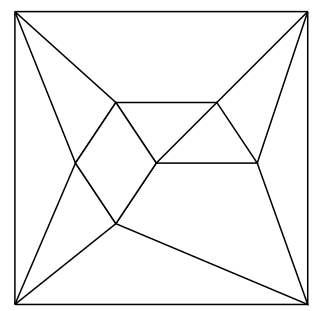

Figure 7: Hyperbolic octahedrite with 10 vertices

Case D1 Let $P_{0} \in \Omega_{3}(\mathscr{P})$ be the hyperbolic octahedrite with ten vertices depicted in Figure 8. Consider the facets of $\mathscr{P}$ adjacent to $P_{0}$ at its faces. One has $f_{2}\left(P_{0}\right)=12$, and hence $f_{3}(\mathscr{P}) \geq 12$. Consider the faces coloured grey in Figure 8: the front face is called $F_{1}$ and the back face, called $F_{2}$, is indicated by the grey arrow.

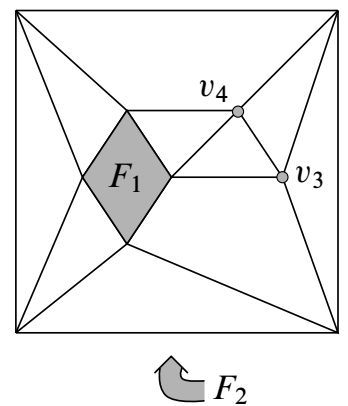

Figure 8: Hyperbolic octahedrite with 10 vertices as a facet of $\mathscr{P}$ and its neighbours

The facets $P_{1}, P_{2} \in \Omega_{3}(\mathscr{P})$ adjacent to $P_{0}$ at $F_{1}$ and $F_{2}$, respectively, contain quadrilaterals among their faces. By Proposition 5, it follows that $\mathrm{f}_{2}\left(P_{i}\right) \geq \mathrm{f}_{2}\left(\mathscr{A}_{4}\right)=10$, $i=1,2$. We shall count all new facets $P^{\prime}$ brought by face adjacency to $P_{i}, i=1,2$.

Observe that no $P^{\prime}$, which does not share an edge with $P_{0}$, can be adjacent simultaneously to $P_{i}$ and $P_{j}, i, j \in\{1,2\}$, at two-faces, since otherwise the $(4,0)$ circuit $P_{1} P_{0} P_{2} P^{\prime}$ appears in contrary to Lemma 6. 
Each facet $P^{\prime}$ that shares an edge with $F_{k}, k=1,2$, is already counted as adjacent to $P_{0}$. The facets $P_{1}$ and $P_{2}$ are already counted as well, by the same reason. Then the total number of new facets coming together with $P_{1}$ and $P_{2}$ is at least $\sum_{i=1}^{2} \mathrm{f}_{2}\left(P_{i}\right)-\sum_{i=1}^{2} \mathrm{f}_{1}\left(F_{i}\right)-2 \geq 2 \cdot 10-2 \cdot 4-2=10$. This implies the estimate $\mathrm{f}_{3}(\mathscr{P}) \geq 12+10=22$.

Consider the facets $P_{i}, i=3,4$, adjacent to $P_{0}$ only at the corresponding circumscribed grey vertices $v_{i}, i=3,4$, in Figure 8 . Then consider the case if $P^{\prime}$ is adjacent to $P_{j}, j \in\{1,2\}$ at a two-face $F^{\prime} \in \Omega_{2}\left(P_{j}\right)$. If there exist a face $\tilde{F}^{\prime} \in \Omega_{2}\left(P_{0}\right)$ such that $F^{\prime} \cap \widetilde{F}^{\prime} \neq \varnothing$, then choose a point $p \in F^{\prime} \cap \tilde{F}^{\prime}$ and use the convexity argument again for the geodesic going through $p$ to $v_{i}$. If $F^{\prime} \cap \widetilde{F}^{\prime}=\varnothing$, then the $(2,1)$ circuit $P_{0} P_{1} P^{\prime}$ appears in contrary to Lemma 6 . Adding up two new facets gives $\mathrm{f}_{3}(\mathscr{P}) \geq 24$. Finally, we count $P_{0}$ itself and arrive at the estimate $f_{3}(\mathscr{P}) \geq 25$.

Case D2 Let $P_{0} \in \Omega_{3}(\mathscr{P})$ be the hyperbolic octahedrite with nine vertices and eleven faces depicted on the right in Figure 6. Consider the facets adjacent to $P_{0}$ at its two-dimensional faces. By counting them, we have $\mathrm{f}_{3}(\mathscr{P}) \geq \mathrm{f}_{2}\left(P_{0}\right)=11$.

Consider the facet $P_{1}$ adjacent to the triangle face $F_{1}$ of $P_{0}$ coloured grey in the center of Figure 9. By Proposition 5, we have $\mathrm{f}_{2}\left(P_{1}\right) \geq \mathrm{f}_{2}\left(\mathscr{A}_{3}\right)=8$. By excluding already counted facets adjacent to $P_{0}$ like in case D1, the facet $P_{1}$ brings new $\mathrm{f}_{2}\left(P_{1}\right)-$ $\mathrm{f}_{1}\left(F_{1}\right)-1 \geq 8-3-1=4$ ones by face adjacency. Then $\mathrm{f}_{3}(\mathscr{P}) \geq 15$. The visible part of the facet $P_{2}$ adjacent to $P_{0}$ at its back face $F_{2}$ is coloured grey in Figure 9. Again, we have $\mathrm{f}_{2}\left(P_{2}\right) \geq \mathrm{f}_{2}\left(\mathscr{A}_{3}\right)=8$. By counting new facets adjacent to $P_{2}$ at faces, it brings another $\mathrm{f}_{2}\left(P_{2}\right)-\mathrm{f}_{1}\left(F_{2}\right)-1 \geq 8-3-1=4$ new ones. Hence $\mathrm{f}_{3}(\mathscr{P}) \geq 19$.

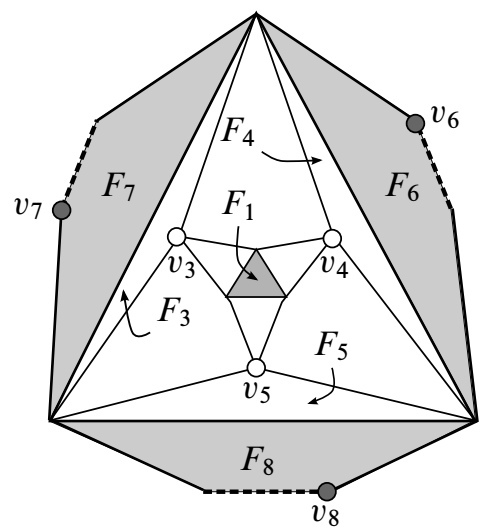

Figure 9: Hyperbolic octahedrite with 9 vertices as a facet of $\mathscr{P}$ and its neighbours (omitted edges are dotted)

The facets $\hat{P}_{k}, k=3,4,5$, adjacent to $P_{0}$ only at the circumscribed hollow vertices $v_{k}, k=3,4,5$, in Figure 9 are different from the already counted ones either by the 
convexity argument or by Lemma 6 , which forbids $(2,1)$ circuits, c.f. the argument of case D1. Thus $\mathrm{f}_{3}(\mathscr{P}) \geq 22$.

Let $\widehat{P}_{k}, k=6,7,8$, be the facets of $\mathscr{P}$ adjacent to $P_{2}$ only at the respective circumscribed grey vertices $v_{k}, k=6,7,8$ in Figure 9. Let the faces of $P_{1}$ and $P_{2}$, that contain a single circumscribed hollow or grey vertex, be $F_{k}, k=3, \ldots, 8$. Finally, let $P(k), k=6,7,8$, denote the facets adjacent to $P_{2}$ at $F_{k}, k=6,7,8$, respectively.

By the convexity argument or by Lemma 6 , similar to D1, the facets $\widehat{P}_{i}, i=6,7,8$ can not coincide with the already counted ones, except for $\hat{P}_{j}, j=3,4,5$ and the facets adjacent only to $P_{1}$.

First consider the case when a facet from $\widehat{P}_{i}, i \in\{6,7,8\}$, coincides with $\widehat{P}_{j}, j \in$ $\{3,4,5\}$. Then

(1) either $\widehat{P}_{i}=\widehat{P}_{j}$ is such that $(i, j) \neq(7,3),(6,4)$ and $(8,5)$, so the $(2,1)$ circuit $\widehat{P}_{j} P(i) P_{0}$ appears;

(2) or $\widehat{P}_{i}=\widehat{P}_{j}$ has $(i, j)=(7,3),(6,4)$, or $(8,5)$, and contains therefore a part of the geodesic going from $v_{i}$ to $v_{j}$ by convexity. Since the edge shared by $F_{i}$ and $F_{j}$ belongs to three facets $P_{0}, P_{2}$ and $P(i)$, then $P(i)$ is adjacent to $P_{0}$ at $F_{j}$ and to $P_{2}$ at $F_{i}$. Hence $P(i)$ contains the vertices $v_{i}, v_{j}$ and the geodesic segment between them as well. Since $P(i)$ and $\widehat{P}_{i}$ have nonintersecting interiors, the two following cases are only possible.

(2.1) The geodesic segment $v_{i} v_{j}$ belongs to a triangle face of $P(i)$ : then $v_{i} v_{j}$ is an edge. Observe that the face $F_{j}$ of $P(i)$ is always a triangle, as in Figure 9, while the face $F_{i}$ is either a triangle or a quadrilateral. Then the edges of $F_{i}$, $F_{j}$ and the edge $v_{i} v_{j}$ constitute a sub-graph in the one-skeleton of $P(i)$. The possible sub-graphs $\tau$ and $\sigma$ depending on the vertex number of $F_{i}$ are depicted in Figure 10. The graph $\tau$ is the one-skeleton of a tetrahedron. The graph $\sigma$ is the one-skeleton of a square pyramid without one vertical edge. By assumption, the facet $P(i)$ is an octahedrite with not more than ten vertices. Such octahedrites are depicted in Figures 6-7, and none of them contains in its one-skeleton a sub-graph combinatorially isomorphic to $\tau$ or $\sigma$.

The case when $P(i)$ is an octahedron still remains. Clearly, its one-skeleton does not contain a sub-graph combinatorially isomorphic to $\tau$. However, it contains a sub-graph isomorphic to $\sigma$. The only possible sub-graph embedding of $\sigma$ into the one-skeleton of an octahedron, up to a symmetry, is given in Figure 11 on the left. But then the face $F_{i}$ of $P_{2}$ correspond to the interior domain $F$ in $P(i)$ coloured grey in Figure 11 on the right. Thus, we arrive at a contradiction with the convexity of facets. 

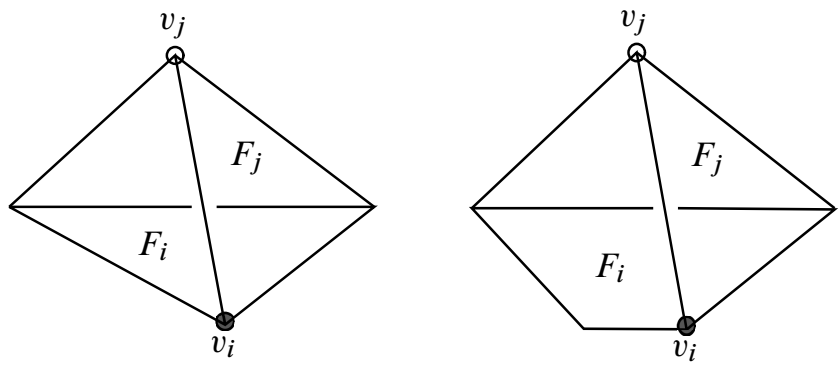

Figure 10: Sub-graphs $\tau$ (on the left) and $\sigma$ (on the right)

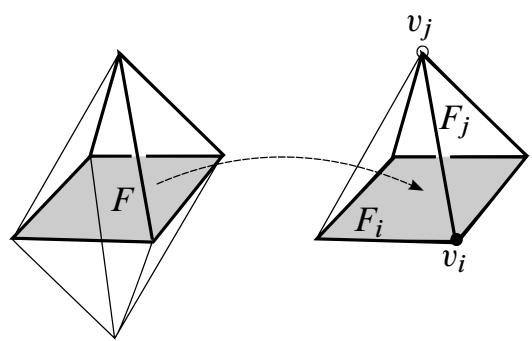

Figure 11: Sub-graph $\sigma$ in an octahedron (on the left) and in the facet $P(i)$ (on the right)
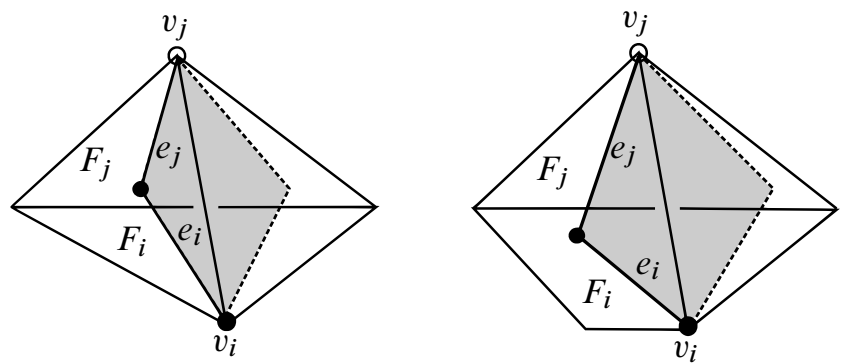

Figure 12: The segment $v_{i} v_{j}$ belongs to a quadrilateral face

(2.2) The geodesic segment $v_{i} v_{j}$ belongs to a quadrilateral face of $P(i)$. The general picture of this case is given in Figure 12. Again two sub-graphs $v$ and $\omega$ arise, as depicted in Figure 13. Such sub-graphs appear at most for the octahedrites as given in Figures 6-7. Observe, that none of them contains in its one-skeleton a sub-graph isomorphic to $v$.

All possible embeddings of $\omega$ into the one-skeleton of each considered octahedrite are given, up to a symmetry, in Figures 14-21. Since the edges $e$ and $e^{\prime}$ belong to a single face as in Figure 12, we arrive at a contradiction, since there is no embedding of $\omega$ with this property. 

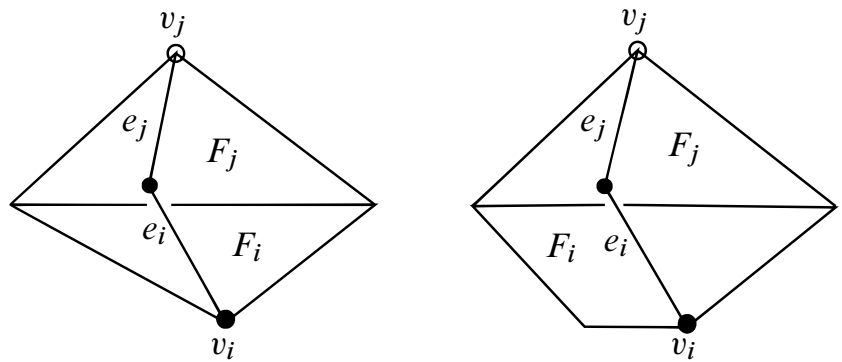

Figure 13: Sub-graphs $v$ (on the left) and $\omega$ (on the right)
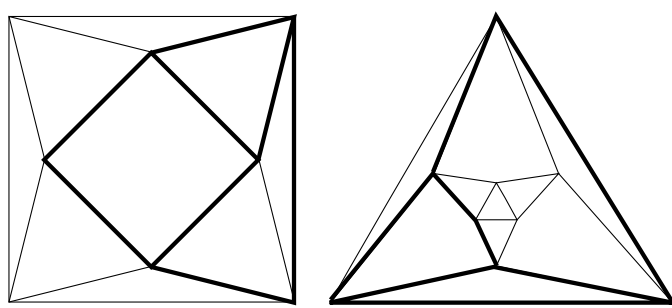

Figure 14: Embeddings of the graph $v$ into octahedrite facets with 8 (left) and 9 (right) vertices

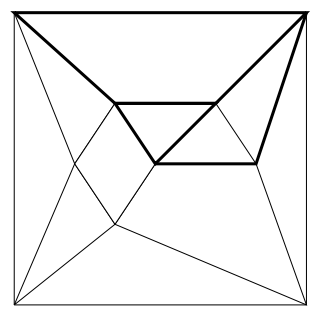

Figure 15: An embedding of the graph $\omega$ into the octahedrite facet with 10 vertices

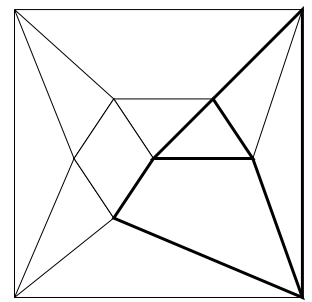

Figure 16: An embedding of the graph $\omega$ into the octahedrite facet with 10 vertices

Finally, consider the case when a facet from $\widehat{P}_{i}, i \in\{6,7,8\}$, coincides with a facet $P^{\prime}$ adjacent only to $P_{1}$ at a two-face. Then the $(4,0)$ circuit $P_{0} P_{1} P^{\prime} P(i)$ arises, in contrary to Lemma 6. 


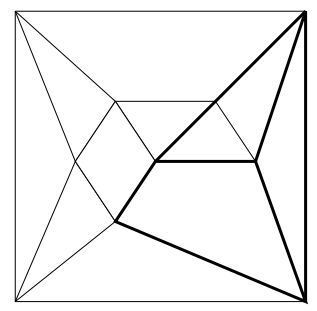

Figure 17: An embedding of the graph $\omega$ into the octahedrite facet with 10 vertices

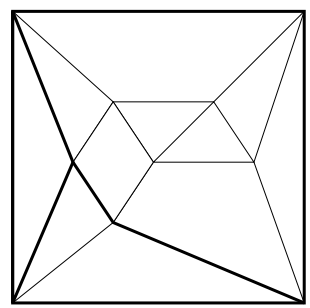

Figure 18: An embedding of the graph $\omega$ into the octahedrite facet with 10 vertices

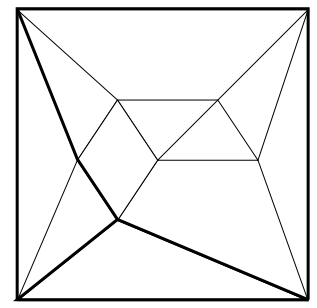

Figure 19: An embedding of the graph $\omega$ into the octahedrite facet with 10 vertices

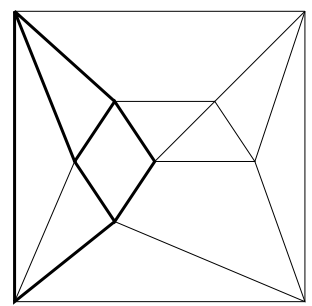

Figure 20: An embedding of the graph $\omega$ into the octahedrite facet with 10 vertices

So the facets $\widehat{P}_{k}, k=6,7,8$, are different from the already counted ones. Adding them up, we obtain $\mathrm{f}_{3}(\mathscr{P}) \geq 22+3=25$. 


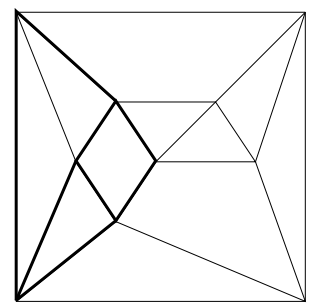

Figure 21: An embedding of the graph $\omega$ into the octahedrite facet with 10 vertices

Case D3 Let $P_{0} \in \Omega_{3}(\mathscr{P})$ be the hyperbolic octahedrite with eight vertices depicted on the left in Figure 6. Observe that this polyhedron is combinatorially isomorphic to $\mathscr{A}_{4}$, and hence isometric to it by Andreev's theorem [2]. Moreover, we suppose that all facets of $\mathscr{P}$ are isometric to $\mathscr{A}_{4}$, since other possible facet types are already considered in Cases D1 and D2.

Consider the facets $P_{k}, k=1,2$, adjacent to the front and the back quadrilateral faces of $P_{0}$. The facets $P_{i}, i=0,1,2$, are depicted together in Figure 22, where $P_{0}$ is coloured grey. We count the facets adjacent to $P_{i}, i=1,2,3$, at faces in Figure 22. Observe that different numbers on the faces shown in Figure 22 correspond to distinct facets of $\mathscr{P}$ adjacent to them. The counting arguments are completely analogous to those of Case C. Hence, we obtain the estimate $\mathrm{f}_{3}(\mathscr{P}) \geq 18$. By taking into account the facets $P_{i}, i=1,2,3$, themselves, it becomes $\mathrm{f}_{3}(\mathscr{P}) \geq 21$.

Consider the facets $\hat{P}_{i}, i=1,2,3,4$, adjacent to $P_{2}$ only at its circumscribed vertices $v_{i}, i=1,2,3,4$ in Figure 22. By analogy with the proof in Case D2, the $\hat{P}_{i}$ 's are different from the already counted ones. Thus, we add four new facets and obtain $\mathrm{f}_{3}(\mathscr{P}) \geq 25$.

Hence, a polytope $\mathscr{P}$ with $\mathrm{f}_{3}(\mathscr{P})=24$ has only octahedral facets and, by the argument from Theorem 3, is isometric to the hyperbolic 24-cell.

\section{A dimension bound for ideal right-angled hyperbolic poly- topes}

Given a combinatorial $n$-dimensional polytope $\mathscr{P}$, define the average number of $\ell$-dimensional faces over $k$-dimensional ones as

$$
\mathrm{f}_{k}^{\ell}(\mathscr{P})=\frac{1}{\mathrm{f}_{k}(\mathscr{P})} \sum_{P \in \Omega_{k}(\mathscr{P})} \mathrm{f}_{\ell}(P) .
$$




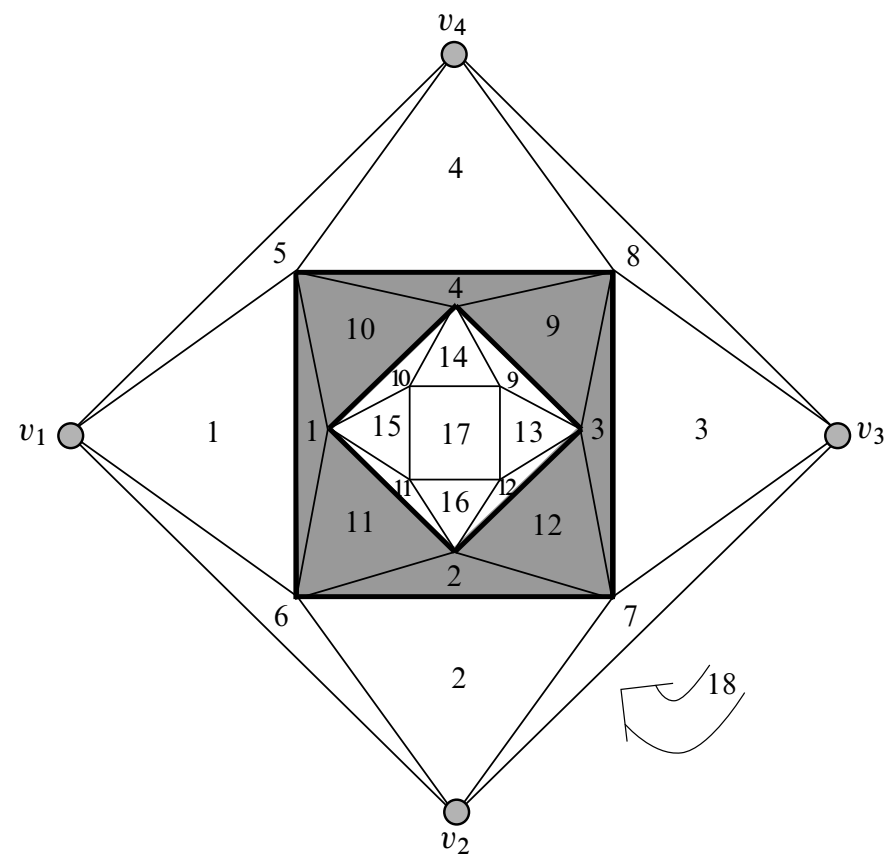

Figure 22: Hyperbolic octahedrite with 8 vertices as a facet of $\mathscr{P}$ and its neighbours

The Nikulin-Khovanskiı̌ inequality (see Khovanskiǔ [7] and Nikulin [8]) applied to the polytope $\mathscr{P}$ which is simple at edges, states that

$$
\mathrm{f}_{k}^{\ell}(\mathscr{P})<\left(\begin{array}{l}
n-\ell \\
n-k
\end{array}\right) \frac{\left(\begin{array}{c}
\left\lfloor\frac{n}{2}\right\rfloor \\
\ell
\end{array}\right)+\left(\begin{array}{c}
\left\lfloor\frac{n+1}{2}\right\rfloor \\
\ell
\end{array}\right)}{\left(\begin{array}{c}
\left\lfloor\frac{n}{2}\right\rfloor \\
k
\end{array}\right)+\left(\begin{array}{c}
\left\lfloor\frac{n+1}{2}\right\rfloor \\
k
\end{array}\right)},
$$

where $\lfloor\circ\rfloor$ means the floor function.

Corollary 7 (of Theorem 4) There are no ideal right-angled hyperbolic polytopes in $\mathbb{H}^{n}$, if $n \geq 7$.

Proof Suppose that $\mathscr{P} \subset \mathbb{H}^{n}$ is an ideal right-angled hyperbolic polytope, $n \geq 4$. Since we have $\mathrm{f}_{4}^{3}(\mathscr{P}) \geq 24$ by Theorem 4 , then (9) implies $n \leq 5$ for $n$ odd and $n \leq 6$ for $n$ even.

\section{References}

[1] E M Andreev, On convex polyhedra in Lobačevskiŭ space, Math. USSR Sb. 10 (1970) $413-440$ 
[2] E M Andreev, On convex polyhedra of finite volume in Lobačevskiŭ space, Math. USSR Sb. 12 (1970) 255-259

[3] HS M Coxeter, Regular polytopes, 3rd edition, Dover Publications, New York (1973) MR0370327

[4] M Deza, M Shtogrin, Octahedrites, Symmetry Cult. Sci. 11 (2000) 27-64 MR2001411

[5] M Deza, MD Sikirić, M Shtogrin, Fullerene-like spheres with faces of negative curvature arXiv:1112.3320

[6] G Dufour, Notes on right-angled Coxeter polyhedra in hyperbolic spaces, Geom. Dedicata 147 (2010) 277-282 MR2660580

[7] A G Khovanskiü, Hyperplane sections of polyhedra, toric varieties and discrete groups in Lobačhevskiŭ space, Funktsional. Anal. i Prilozhen. 20 (1986) 50-61, 96 MR831049

[8] V V Nikulin, On the classification of arithmetic groups generated by reflections in Lobačevskiŭ spaces, Izv. Akad. Nauk SSSR Ser. Mat. 45 (1981) 113-142, 240 MR607579

[9] L Potyagailo, E Vinberg, On right-angled reflection groups in hyperbolic spaces, Comment. Math. Helv. 80 (2005) 63-73 MR2130566

[10] J G Ratcliffe, S T Tschantz, The volume spectrum of hyperbolic 4-manifolds, Experiment. Math. 9 (2000) 101-125 MR1758804

[11] E B Vinberg, editor, Geometry II: Spaces of constant curvature, Encyclopaedia of Mathematical Sciences 29, Springer, Berlin (1993) MR1254931 A translation of Geometriya. II, Akad. Nauk SSSR, Vsesoyuz. Inst. Nauchn. i Tekhn. Inform., Moscow, 1988, Translation by V V Minakhin

[12] T Zehrt, The covolume of discrete subgroups of Iso( $\left.\mathbb{H}^{2 m}\right)$, Discrete Math. 309 (2009) 2284-2291 MR2510356

[13] G M Ziegler, Lectures on polytopes, Graduate Texts in Mathematics 152, Springer, New York (1995) MR1311028

Department of Mathematics, University of Fribourg

Chemin du Musée 23, CH-1700 Fribourg, Switzerland

kolpakov.alexander@gmail.com

Received: 22 June 2012 Revised: 11 July 2012 